\title{
Fe de erratas: Registro colombiano de falla cardiaca (RECOLFACA): metodología y datos preliminaries. Actualización septiembre 2021 [Revista Colombiana de Cardiología. 2021;28(3):217-230]
}

Erratum: Colombian heart failure registry (RECOLFACA): methodology and preliminary data. Update Septembre 2021 [Revista Colombiana de Cardiología. 2021;28(3):217-230]

\section{Juan E. Gómez-Mesa ${ }^{1,4 *}$, Clara I. Saldarriaga ${ }^{2,4}$, Luis E. Echeverría ${ }^{3,4}$, Paula Luna ${ }^{4}$ y} Grupo Investigador Recolfaca

\#Véase anexo de lista del Grupo Investigador RECOLFACA

${ }^{1}$ Servicio de Cardiología, Fundación Valle del Lili, Cali; ${ }^{2}$ Servicio de Cardiología, Clínica Cardio VID, Medellín; ${ }^{3}$ Servicio de Cardiología, Fundación Cardiovascular de Colombia, Bucaramanga; ${ }^{4}$ Sociedad Colombiana de Cardiología, Bogotá. Colombia

En el artículo publicado anteriormente, Registro colombiano de falla cardiaca (RECOLFACA): metodología $y$ datos preliminaries. [Revista Colombiana de Cardiología. 2021;28(3):217-230] se ha detectado algunos errores:

\section{Características clínicas}

La etiología más frecuente de la falla cardiaca fue la isquémica $(43.99 \%)$, seguida de la hipertensiva (31.80\%). El $11.83 \%$ de los pacientes tenían una clase funcional NYHA I, el $53.60 \%$ tenían clase II, el 29.87\% clase III y el $4.71 \%$ clase IV. La clasificación ACC/AHA más común fue C $(94.54 \%)$. El $73.54 \%$ de los pacientes tenían una FEVI reducida ( $<40 \%)$, el $10.33 \%$ en rango intermedio (40-50\%) y el $16.13 \%$ preservada (> 50\%).

El 22.39\% tenían algún tipo de dispositivo implantable (cardiodesfibrilador implantable [CDI] el $9.69 \%$ y resincronizador más CDI el 5.02\%) (Tabla 2).

Se corrige el Anexo de miembros del grupo Investigador RECOLFACA y, por último, también la figura 1 está actualizada.

\section{Correspondencia:}

*Juan E. Gómez-Mesa

E-mail: juanestebang@yahoo.com
Disponible en internet: $27-10-2021$ Rev Colomb Cardiol. 2021;28(6):669-672 www.rccardiologia.com 0120-5633 / @ 2021 Sociedad Colombiana de Cardiología y Cirugía Cardiovascular. Publicado por Permanyer. Este es un artículo open access bajo la licencia CC BY-NC-ND (http://creativecommons.org/licenses/by-nc-nd/4.0/). 


\section{Miembros del Grupo Investigador RECOLFACA}

\begin{tabular}{|c|c|c|c|}
\hline $\begin{array}{l}\text { INVESTIGADOR } \\
\text { PRINCIPAL }\end{array}$ & INSTITUCIÓN & $\begin{array}{l}\text { INVESTIGADOR } \\
\text { PRINCIPAL }\end{array}$ & INSTITUCIÓN \\
\hline Alberto Cadena & $\begin{array}{l}\text { Clínica de la Costa. Barranquilla, } \\
\text { Colombia }\end{array}$ & Erika Martínez & $\begin{array}{l}\text { IPS Salud Social. Barranquilla, } \\
\text { Colombia }\end{array}$ \\
\hline $\begin{array}{l}\text { Alejandro David Ochoa } \\
\text { Morón }\end{array}$ & $\begin{array}{l}\text { Instituto Cardiovascular del Cesar } \\
\text { ICVC. Cesar, Colombia }\end{array}$ & Fernán Mendoza Beltrán & $\begin{array}{l}\text { Fundación Clínica Shaio. Bogotá, } \\
\text { Colombia }\end{array}$ \\
\hline $\begin{array}{l}\text { Alejandro Posada } \\
\text { Bastidas }\end{array}$ & $\begin{array}{l}\text { Centro Cardiovascular Somer In } \\
\text { Care. Rionegro, Colombia }\end{array}$ & Fernando Manzur Jatin & $\begin{array}{l}\text { Centro Diagnóstico Cardiológico. } \\
\text { Cartagena, Colombia }\end{array}$ \\
\hline Alex Rivera Toquica & $\begin{array}{l}\text { Clínica los Rosales. Centro } \\
\text { Médico para el Corazón. Pereira, } \\
\text { Colombia }\end{array}$ & Fernando Rivera Toquica & $\begin{array}{l}\text { Sanidad Policía Nacional } \\
\text { Seccional Risaralda. Pereira, } \\
\text { Colombia }\end{array}$ \\
\hline Alexis Llamas & $\begin{array}{l}\text { Clínica Las Américas. Medellín, } \\
\text { Colombia }\end{array}$ & $\begin{array}{l}\text { Gary Andres Baquero } \\
\text { Lozano }\end{array}$ & $\begin{array}{l}\text { Hospital Santa Clara. Bogotá, } \\
\text { Colombia }\end{array}$ \\
\hline Alfonso Muñoz Velásquez & $\begin{array}{l}\text { Institución Clínica Iberoamérica. } \\
\text { Barranquilla, Colombia }\end{array}$ & Guillermo Trout Guardiola & $\begin{array}{l}\text { Group Salud IPS. Santa Marta, } \\
\text { Colombia }\end{array}$ \\
\hline $\begin{array}{l}\text { Álvaro Hernán Rodríguez } \\
\text { Cerón }\end{array}$ & $\begin{array}{l}\text { Hospital Cardiovascular de } \\
\text { Cundinamarca. Soacha, Colombia }\end{array}$ & Gustavo Moreno Silgado & $\begin{array}{l}\text { Hospicardio. Montería, } \\
\text { Colombia }\end{array}$ \\
\hline Andres F. Buitrago & $\begin{array}{l}\text { Fundación Santa Fe. Bogotá, } \\
\text { Colombia }\end{array}$ & $\begin{array}{l}\text { Hugo Ernesto Osorio } \\
\text { Carmona }\end{array}$ & $\begin{array}{l}\text { Clínica Medilaser Neiva. Neiva, } \\
\text { Colombia }\end{array}$ \\
\hline $\begin{array}{l}\text { Ángel Alberto García } \\
\text { Peña }\end{array}$ & $\begin{array}{l}\text { Hospital Universitario San } \\
\text { Ignacio. Bogotá, Colombia }\end{array}$ & Jannes Buelvas Herazo & $\begin{array}{l}\text { Cardiodiagnóstico SA. } \\
\text { Barranquilla, Colombia }\end{array}$ \\
\hline $\begin{array}{l}\text { Argemiro Ramon Martínez } \\
\text { Carvajal }\end{array}$ & $\begin{array}{l}\text { Hospital San Antonio de } \\
\text { Roldanillo. Valle del Cauca, } \\
\text { Colombia }\end{array}$ & $\begin{array}{l}\text { Jorge Alberto Sandoval } \\
\text { Luna }\end{array}$ & $\begin{array}{l}\text { Cardiología Siglo XXI. Ibagué, } \\
\text { Colombia }\end{array}$ \\
\hline $\begin{array}{l}\text { Armando Alcalá } \\
\text { Hernandez }\end{array}$ & $\begin{array}{l}\text { Centro de Cardiología } \\
\text { Procardiocaribe S.A.S. }\end{array}$ & $\begin{array}{l}\text { Jose Ignacio Mosquera } \\
\text { Jiménez }\end{array}$ & $\begin{array}{l}\text { Unidad Cardiológica del Cauca } \\
\text { Única S.A.S. Popayán, Colombia }\end{array}$ \\
\hline & Barranquilla, Colombia & José Luis Accini Mendoza & $\begin{array}{l}\text { Centro Científico Asistencial SAS. } \\
\text { Barranquilla. Colombia }\end{array}$ \\
\hline Balkis Rolong & $\begin{array}{l}\text { Cardiología Integral. Barranquilla, } \\
\text { Colombia }\end{array}$ & Juan Alberto Cerón & Unimedic IPS. Pasto, Colombia \\
\hline $\begin{array}{l}\text { Carlos Alberto Rentería } \\
\text { Asprilla }\end{array}$ & $\begin{array}{l}\text { Cardiodiagnóstico del Chocó. } \\
\text { Choco, Colombia }\end{array}$ & Juan Camilo García & $\begin{array}{l}\text { Hospital Universidad del Norte. } \\
\text { Barranquilla, Colombia }\end{array}$ \\
\hline $\begin{array}{l}\text { Carlos Andres Arias } \\
\text { Barrera }\end{array}$ & $\begin{array}{l}\text { Clínica Universitaria Colombia } \\
\text { Colsanitas. Bogotá, Colombia }\end{array}$ & $\begin{array}{l}\text { Juan Carlos Ortega } \\
\text { Madariaga }\end{array}$ & $\begin{array}{l}\text { Hospital Universitario Erasmo } \\
\text { Meoz. Cúcuta, Colombia }\end{array}$ \\
\hline $\begin{array}{l}\text { Carlos Andres Plata } \\
\text { Mosquera }\end{array}$ & $\begin{array}{l}\text { Dime Clínica Neuro } \\
\text { Cardiovascular. Cali, Colombia }\end{array}$ & $\begin{array}{l}\text { Juan David López Ponce } \\
\text { de León }\end{array}$ & $\begin{array}{l}\text { Fundación Valle de Lili. Cali, } \\
\text { Colombia }\end{array}$ \\
\hline $\begin{array}{l}\text { Clara Ines Saldarriaga } \\
\text { Giraldo }\end{array}$ & $\begin{array}{l}\text { Clínica CardioVid. Medellín, } \\
\text { Colombia. }\end{array}$ & $\begin{array}{l}\text { Juan Fernando Carvajal } \\
\text { Estupiñán }\end{array}$ & $\begin{array}{l}\text { Instituto del Corazón de } \\
\text { Bucaramanga. Bucaramanga, } \\
\text { Colombia }\end{array}$ \\
\hline Claudıa Vıctorıa Anchıque & $\begin{array}{l}\text { Mediagnostica. Duitama, } \\
\text { Colombia }\end{array}$ & Julián Rodrigo Lugo Peña & Clínicos IPS. Bogotá, Colombia \\
\hline $\begin{array}{l}\text { Diego Hernán Hoyos } \\
\text { Ballesteros }\end{array}$ & $\begin{array}{l}\text { Centro Cardiovascular \& Diabetes } \\
\text { MASSALUD. Armenia, Colombia }\end{array}$ & Julian Vanegas Eljach & $\begin{array}{l}\text { Clínica Avidanti. Manizales, } \\
\text { Colombia }\end{array}$ \\
\hline $\begin{array}{l}\text { Edgar Eduardo Castro } \\
\text { Osorio }\end{array}$ & $\begin{array}{l}\text { Clínica San Marcel - Confa. } \\
\text { Manizales, Colombia }\end{array}$ & $\begin{array}{l}\text { Lisbeth Natalia Morales } \\
\text { Rodríguez }\end{array}$ & Clínica Medilaser. Tunja, Colombia \\
\hline $\begin{array}{l}\text { Eduardo José Echeverri } \\
\text { Navarrete }\end{array}$ & $\begin{array}{l}\text { Clínica De Occidente. Cali, } \\
\text { Colombia }\end{array}$ & $\begin{array}{l}\text { Luis Eduardo Echeverría } \\
\text { Correa }\end{array}$ & $\begin{array}{l}\text { Fundación Cardiovascular de } \\
\text { Colombia. Floridablanca, } \\
\text { Colombia }\end{array}$ \\
\hline $\begin{array}{l}\text { Elkin Giovanni Ramírez } \\
\text { Puentes }\end{array}$ & $\begin{array}{l}\text { Sociedad Cardiológica } \\
\text { Colombiana S.A.S. Villavicencio, } \\
\text { Colombia }\end{array}$ & Luis Manuel Ávila Barros & $\begin{array}{l}\text { Clínica Riohacha. Riohacha, } \\
\text { Colombia }\end{array}$ \\
\hline
\end{tabular}




\begin{tabular}{|c|c|}
\hline $\begin{array}{l}\text { INVESTIGADOR } \\
\text { PRINCIPAL }\end{array}$ & INSTITUCIÓN \\
\hline Luis Silva Díaz Granados & Clínica Chía. Chía, Colombia \\
\hline $\begin{array}{l}\text { Luz Clemencia Zarate } \\
\text { Correa }\end{array}$ & $\begin{array}{l}\text { Clínica Imbanaco, Hospital } \\
\text { Universitario del Valle. Cali, } \\
\text { Colombia }\end{array}$ \\
\hline $\begin{array}{l}\text { Marco Antonio De León } \\
\text { Espitia }\end{array}$ & $\begin{array}{l}\text { Cardio Care Colombia SAS. } \\
\text { Montería, Colombia. }\end{array}$ \\
\hline $\begin{array}{l}\text { Mario Hernán Zarama } \\
\text { Márquez }\end{array}$ & $\begin{array}{l}\text { Clínica Nuestra Señora de Fátima. } \\
\text { Pasto, Colombia. }\end{array}$ \\
\hline Nelly Velásquez López & $\begin{array}{l}\text { Clínica Medellín. Medellín, } \\
\text { Colombia }\end{array}$ \\
\hline $\begin{array}{l}\text { Nelson Adolfo López } \\
\text { Garzón }\end{array}$ & $\begin{array}{l}\text { Hospital Universitario San José. } \\
\text { Popayán, Colombia }\end{array}$ \\
\hline $\begin{array}{l}\text { Nelson Eduardo Murillo } \\
\text { Benítez }\end{array}$ & $\begin{array}{l}\text { Angiografía de Occidente. Cali, } \\
\text { Colombia }\end{array}$ \\
\hline $\begin{array}{l}\text { Oscar Alfredo Pacheco } \\
\text { Jiménez }\end{array}$ & $\begin{array}{l}\text { Hospital Militar Central. Bogotá, } \\
\text { Colombia }\end{array}$ \\
\hline Oscar Sveins Rincón Peña & $\begin{array}{l}\text { Unidad de Diagnóstico } \\
\text { Cardiovascular Sveins. Duitama, } \\
\text { Colombia }\end{array}$ \\
\hline Patricia Rodríguez Gómez & $\begin{array}{l}\text { Unidad Cardiológica de } \\
\text { Cartagena. Cartagena, Colombia }\end{array}$ \\
\hline $\begin{array}{l}\text { Rafael Ignacio } \\
\text { Bustamante Urzola }\end{array}$ & $\begin{array}{l}\text { Rafael Bustamante y Compañía } \\
\text { Ltda. Leticia, Colombia }\end{array}$ \\
\hline Ricardo Gómez Palau & Clínica Amiga. Cali, Colombia \\
\hline Rolando Palacio & $\begin{array}{l}\text { Clínica Renacer. Riohacha, } \\
\text { Colombia }\end{array}$ \\
\hline $\begin{array}{l}\text { Sebastián Campbell } \\
\text { Quintero }\end{array}$ & $\begin{array}{l}\text { Clínica Mediláser. Florencia, } \\
\text { Colombia }\end{array}$ \\
\hline Silfriedo Arrieta González & $\begin{array}{l}\text { Hospital Universitario de } \\
\text { Sincelejo. Sincelejo, Colombia }\end{array}$ \\
\hline Viviana Quintero Yepes & $\begin{array}{l}\text { Clínica El Rosario. Medellín, } \\
\text { Colombia }\end{array}$ \\
\hline
\end{tabular}

Véase contenido relacionado en DOI: 10.24875/RCCAR.M21000021 


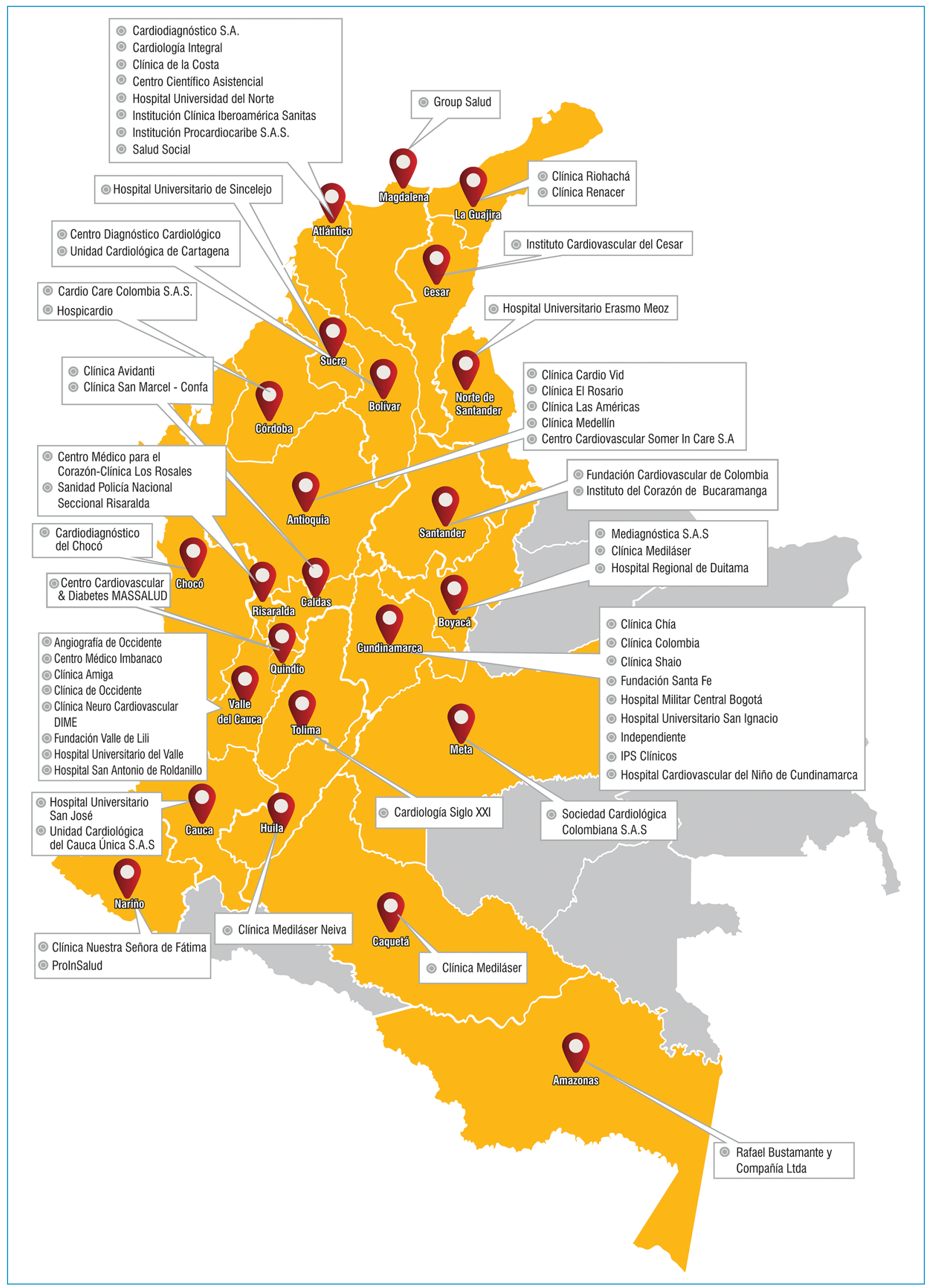

Figura 1. Instituciones participantes en el RECOLFACA por departamentos. 